\title{
SERVO DESIGN FOR HIGH-TPI HARD DISK DRIVES USING A DELAY-ACCOMMODATING STATE ESTIMATOR
}

\author{
Young-Hoon Kim*, Sang-Hoon Chu**, S. W. Kang*, D. H. Oh*, Y. S. Han*, T. Y. Hwang* \\ * Storage Lab., Samsung Advanced Institute of Technology, \\ San 14-1, Nongseo-ri, Giheung-eup, Yongin-si, Gyeonggi-do, KOREA \\ e-mail:younghoon@sait.samsung.co.kr \\ ** HDD R\&D center, Samsung Information Systems \\ 75 west plumeria drive, San Jose, CA 95134, U.S.A. \\ e-mail:shchu@sisa.samsung.com
}

\begin{abstract}
A servo design method is presented for high track-density hard disk drives, in which the plant time delay, mainly due to the processor computation time, is taken into account. The key idea is to incorporate the delay model into the output equation of the state-space representation for the plant model. The application results confirm that the proposed method is very effective in realizing a high-bandwidth servo system.
\end{abstract}

\section{INTRODUCTION}

As the track density (represented by track per inch, or TPI) of computer disk drives becomes higher and higher, it is of increasing importance for servo designers to make the best of known information, rather than consider those not modeled as simply "uncertainties" or "disturbances". The computational delay is one good example, which we deal with in this paper.

Prior works on (computational) delay compensation include the traditional technique of using an input-delayed model as in [1]. This model is utilized to make a more reliable state estimation. The delay, however, is still there in the state feedback law. This problem can be resolved by modifying the feedback law to include the one-sample delayed control signal as in [1]. A similar method is developed in [2] for discrete-equivalent design. A good survey on this subject can be found in [4].

This paper proposes a systematic technique for state-space servo control design, which effectively accommodates the transport delay into the control system. The experimental results based on a commercial hard drive are provided as well as some simulation results. They show that the proposed method effectively helps the stability margin by increasing the phase and gain margin of the system by $3-4^{\circ}$ and $0.3 \mathrm{~dB}$ respectively.

Notation: The sampling time and the delay time will be denoted by $T_{s}$ and $T_{d}$, respectively. A bracket is used to represent a discrete-time signal, e.g. $x[k]$.

\section{MODELING OF TIME DELAY}

In the continuous-time, transfer-function framework, the presence of computation delay $T_{d}$ can be effectively modeled by putting an ideal transport-delay element, $\exp \left(-T_{d} s\right)$. In the case of the state-space formalism, in fact, this delay term may be put either at the control input stage in a state equation (input-delayed model) or at the output stage in the output equation (output-delayed model).

\subsection{Input Delayed Model}

The following state-space model is obtained by assuming the delay $T_{d}$ as being present at the control input stage:

$$
\begin{aligned}
& \dot{x}(t)=A_{p} x(t)+B_{p} u\left(t-T_{d}\right) \\
& y(t)=C x(t)
\end{aligned}
$$

Here, $x(t), y(t)$, and $u(t)$ are the state, the output, and the control, respectively. From this input-delayed plant model, the following discrete-time model is generated [1]:

$$
\begin{aligned}
x[k+1] & =A x[k]+B_{0} u[k]+B_{1} u[k-1] \\
y[k] & =C x[k]
\end{aligned}
$$

It is remarked here that the state at the next sample is affected not only by the control value at the current sample but also by that at the last sample.

\subsection{Output-Delayed Model}

This model considers the output of the system as being the $T_{d}$-delayed version of the normal output, $C x(t)$ :

$$
\begin{aligned}
& \dot{x}(t)=A_{p} x(t)+B_{p} u(t) \\
& y(t)=C_{p} x\left(t-T_{d}\right)
\end{aligned}
$$

It can be shown that this leads to the discretization of the following form:

$$
\begin{aligned}
x[k+1] & =A x[k]+B u[k] \\
y[k] & =C x[k]+D u[k-1],
\end{aligned}
$$

where $(A, B)$ are determined as done usually, while 
$(C, D)$ are defined as

$$
C \triangleq C_{p} e^{-T_{d} A_{e}}, \quad D \triangleq-C_{p} e^{-T_{d} A_{e}} \int_{0}^{T_{d}} e^{\lambda A_{e}} B_{p} d \lambda .
$$

Note here that the state evolution equation does not involve the delay under this output-delay formulation.

\section{DESIGN OF DAE'S}

Two types of estimators can be designed based on the two delay-present model representations presented in the last section. Because of space limitation, only the second type (to be called "delay-accommodating estimator (DAE) hereafter), based on the output-delay model, will be presented.

Applying the standard current state estimator equation to the discrete-time plant given by (2) and (3) to get

$$
\begin{aligned}
\hat{x}[k] & =\bar{x}[k]+L(y[k]-\bar{y}[k]) \\
\bar{x}[k+1] & =A x[k]+B u[k]
\end{aligned}
$$

where the variable $\bar{y}[k]$ can be pre-calculated in the stage of state prediction according to

$$
\bar{y}[k+1]=C x[k+1]+D u[k]
$$

Since we have the ideal state equation as shown in (2) the delay-free state feedback law can be implemented by solely using the state estimate in (4). Thereby, accommodation of time delay is automatically achieved.

\section{SIMULATIONS AND EXPERIMENTS}

The proposed design method was applied to an $80 \mathrm{~GB}$ HDD (Samsung Electronics, Co. Ltd). The delay-accommodating estimator as presented was implemented and compared with the conventional state feedback controller, in which the computation delay is considered only as in [1]. As seen in Fig. 1, the use of DAE significantly recovers the both the phase and gain margin as compared to the conventional controller partially accounting for time delay.

This reduction of phase margin translates to the decrease of the error sensitivity peaking as shown in Fig. 2 . This effectively attenuates the amplification ratio of disk-mode related disturbances. The experimental results confirm that the DAE is practically useful in achieving high-bandwidth servo systems required for high-TPI HDD's.

\section{CONCLUSION}

This paper considered an efficient method to compensate for the presence of computational delay in the digital servo control system for hard disk drives. The best advantage of the proposed control method is that it improves the nominal stability margin without heavily modifying the existing state-space control system; thereby, leading to an increased bandwidth, or, better error rejection capability. The DAE method is in fact shown to be very effective also in track-seeking

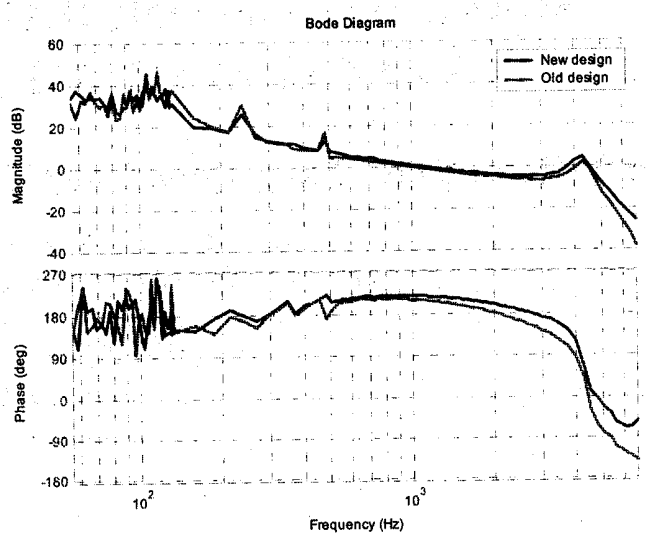

Fig. 1 Comparison of open-loop transfer functions.

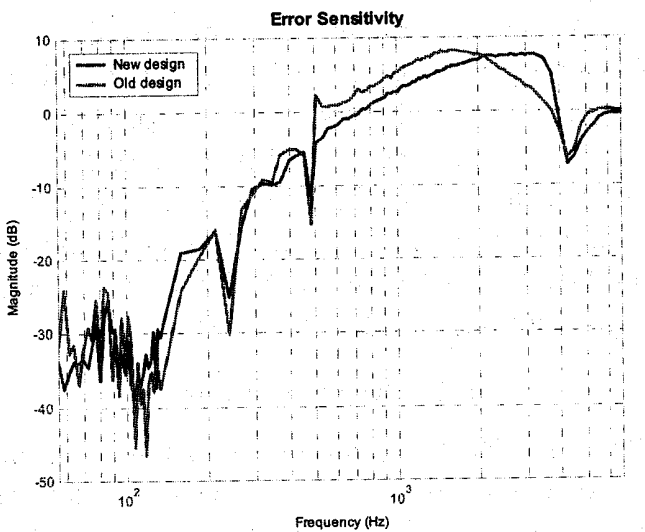

Fig. 2 Comparison of error rejection capability: Old and DAE design.

controllers, although the results are not presented in this paper.

\section{ACKNOWLEDGEMENT}

The authors would like to thank Storage Division, Samsung Electronics, Ltd., as well as Samsung Information System America, Inc. for their valuable help and continued support for this joint project.

\section{REFERENCES}

[1] G.F. Franklin, J.D. Powell, and M.L. Workman, Digital Control of Dynamic Systems, Addison Wesley., 2nd Ed, 1990.

[2] K. S. Rattan, "Compensating for Computational Delay in Digital Equivalent of Continuous Control Systems," IEEE Trans. on Automatic Control, Vol.34, No.8, 1989.

[3] T. Mita, "Optimal Digital Feedback Control Systems Counting Computation Time of Control Laws," IEEE Trans. on Automatic Control, Vol.AC-30, No.6, 1985.

[4] H. Hanselman, "Implementation of Digital Controllers," Automatica., Vol.23, No.1, 1987. 